\title{
Discriminación Étnica En Los Tribunales Y En La Prensa De La Región De La Araucanía En Chile ${ }^{1}$
}

\section{Ethnic Discrimination In The Courts And In The Press In The Region Of La Araucania In Chile}

Dr. Carlos Del Valle Rojas ${ }^{1}$, Dr. Claudio Maldonado Rivera², Mg. Geraldine Iturra Cuevas $^{3}$, Mg. Stefanie Pacheco Pailahual ${ }^{4}$, Mg. Carlos Sáez Figueroa ${ }^{5}$, Mg. Cristian Sanhueza Roa ${ }^{6}$

\footnotetext{
1 - Dr. Carlos Del Valle Rojas - Investigador Proyecto Fondecyt 1120904, Facultad de Educación, Ciencias Sociales y Humanidades Universidad de La Frontera, Temuco, Chile

2 - Dr. Claudio Maldonado Rivera - Tesista Proyecto Fondecyt 1120904, Facultad de Educación, Ciencias Sociales y Humanidades Universidad de La Frontera, Temuco, Chile

3 - Mg. Geraldine Iturra Cuevas - Tesista Proyecto Fondecyt 1120904, Facultad de Educación, Ciencias Sociales y Humanidades Universidad de La Frontera, Temuco, Chile

4 - Mg. Stefanie Pacheco Pailahual - Tesista Proyecto Fondecyt 1120904, Facultad de Educación, Ciencias Sociales y Humanidades Universidad de La Frontera, Temuco, Chile

5 - Mg. Carlos Sáez Figueroa - Tesista Proyecto Fondecyt 1120904, Facultad de Educación, Ciencias Sociales y Humanidades - Universidad de La Frontera, Temuco, Chile

6 - Mg. Cristian Sanhueza Roa - Tesista Proyecto Fondecyt 1120904, Facultad de Educación, Ciencias Sociales y Humanidades - Universidad de La Frontera, Temuco, Chile
}

\footnotetext{
${ }^{1}$ Este trabajo corresponde a una síntesis de los resultados finales del Proyecto de Investigación titulado: "Medios de comunicación y poder: discursos de la Prensa y de sujetos adultos de la región de La Araucanía sobre justicia/injusticia en torno al Conflicto Estado-Nación y Pueblo Mapuche", financiado por el Fondo Nacional de Desarrollo Científico y Tecnológico, FONDECYT-Chile, número 1120904.
} 


\section{Introducción}

Después de años de estudio de la prensa, juicios y especialmente sentencias en los tribunales de la región de La Araucanía, sumado desde luego a la discusiones teóricas correspondientes, me asisten dos convicciones: la imposibilidad ontológica y epistémica de la justicia, y que la tan necesaria como reclamada independencia institucional y formal de los tribunales es menor que la evidente dependencia de las ideologías de las palabras en las sentencias. La tragedia en los tribunales es doble: su exceso de regulación desde el derecho nunca producirá más justicia y su prisión ideológica permanente mediante las palabras que sentencian y devienen en estereotipos, prejuicios y discriminación.

En este sentido, la sentencia de la Corte Interamericana de Derechos Humanos del 17 de mayo de 2014, a favor de un grupo de dirigentes y activistas del pueblo mapuche, en contra del Estado de Chile (contra el cual se falla por violación a los derechos de los imputados), constituye un hito histórico, al menos en los siguientes sentidos: se reconoce el estatus de los estudios y análisis del discurso (en este caso, de las palabras que son sentencias y devienen estereotipos, prejuicios y discriminación) como pruebas válidas, al menos, por ahora, en los tribunales internacionales; valora el rol del análisis de las discursividades como una forma productiva de crítica social con resultados concretos frente a la supuesta "improductividad" de este tipo de análisis; considera la naturaleza compleja de la discriminación, especialmente desde el lenguaje; supera la comprensión aséptica de los tribunales dedicados sólo a la inobjetable gestión y administración de la justicia-, porque los reconoce como productores de decisiones que reproducen las ideologías propias del contexto sociocultural y político al cual pertenecen sus productores (jueces); y permite romper con un tipo de estrategia histórica y hegemónica desde los tribunales, basada en el uso de la diferencia como una forma de exclusión discriminatoria.

\section{Antecedentes}

En síntesis, el peritaje realizado (Del Valle, 2013), muestra tres estrategias empleadas por los tribunales: el uso de expresiones propias de los medios de comunicación, con fuertes cargas valorativas, tanto políticas como morales; la reproducción de estereotipos y prejuicios sociales y culturales sobre las comunidades mapuche, siempre desfavorables a ellas; y la abierta vulneración de derechos mediante el uso de razonamientos discriminatorios. 
En la sentencia se considera este peritaje como base de la "Alegada utilización de estereotipos y prejuicios sociales en las sentencias penales internas" (p. 77); destacando en este caso particular que: "[se] hizo un análisis del 'discurso jurídico-judicial' con el fin de determinar la eventual 'existencia de estereotipos, prejuicios y discriminación en las sentencias penales' de las presuntas víctimas de este caso. Al respecto, [se] concluyó que las sentencias 'utilizan expresiones discursivas cuya carga valorativa, moral y/o política, denota la aceptación y reproducción de estereotipos que incluyen fuertes prejuicios sociales y culturales contras las comunidades mapuche y elementos valorativos en pro de la parte acusadora'. [se] indicó que 'una parte importante de la argumentación jurídica' de dichas decisiones judiciales se desprende de 'estereotipos y prejuicios que recaen nocivamente sobre estas comunidades, $[\ldots]$ sin que se desprenda de hechos probados en el proceso'. Además, [se] sostuvo que 'en diversos extractos de las sentencias $[\ldots$ se $]$ usa[n] argumentos discriminatorios hacia las comunidades mapuche' $y$ que 'en diversas ocasiones se sustentan decisiones jurídicas perjudiciales para miembros o dirigentes mapuche en cadenas de razonamientos que se sustentan, a su vez, en expresiones discriminatorias, estereotipos o prejuicios preconcebidos, en relación al caso examinado'. [Se] analizó distintos extractos de las sentencias internas que [se] considera 'dejan en evidencia' tal 'asimilación de estereotipos y prejuicios y el empleo recurrente de razonamientos discriminatorios' por parte de los tribunales internos.” (p. 78).

En consecuencia con estos y otros argumentos, la Corte declaró por unanimidad que “El Estado violó el principio de igualdad y no discriminación y el derecho a la igual protección de la ley, consagrados en el artículo 24 de la Convención Americana sobre Derechos Humanos” (p. 151).

2. Desarrollo: La Relación EstadoNación Y Pueblo Mapuche

\subsection{El Discurso De La Prensa: El Discurso Del Periódico Mapuche Azkintuwe}

En el discurso de Azkintuwe existe un fenómeno que opera por negatividad, oposición y/o contradicción respecto a los dispositivos por los cuales la colonialidad ha sido ejercida y se ejerce aún sobre el Pueblo Mapuche. Hemos definido esta estrategia discursiva por medio del término "Decolonalidad como Antagonismo", apuntando a los discursos que explicitan una lógica significacional-confrontacional ante las prácticas y discursos que desde la autoridad instalan la colonialidad como mecanismo de clasificación y jerarquización de la población (Maldonado, 2014). 
Esta estrategia opera como rechazo explícito al modus operandi de la colonialidad. En el caso concreto de la tematización abordada referida a las prácticas ejercidas por el Estado-nación chileno y el sistema jurídico-judicial en casos que involucran detenciones y enjuiciamiento a personas mapuches en el marco del conflicto la justicia como parte de la colonialidad es permanentemente enfrentada y resignificada por oposición. Funciona como una dialéctica negativa, sin embargo no es suficiente para proyectar la decolonialidad como praxis por la liberación. Queda encerrada en una lógica oposicional que dentro de un esquema de relaciones interculturales asimétricas no aporta lo suficiente para concretar un proyecto de liberación. Ingresa a un juego ad infinitum en que el sistema es cuestionado, pero no transformado.

Una segunda estrategia, complementaria a la anterior, es aquella que hemos denominado "Decolonialidad por Autonomía", la cual abre posibilidades de reflexión que nos parecen son más vinculantes y pertinentes a la decolonialidad como praxis y proyecto de liberación (Maldonado, 2014). Mientras la estrategia "Decolonialidad como Antagonismo" funciona como crítica a la colonialidad desde la negación de la misma, la "Decolonialidad por Autonomía" es la proyección de la exterioridad - del sujeto subalternizadocomo horizonte legítimo y alternativo de ser, en tanto afirma la mismidad para dar vuelo a una utopística fundada en múltiples posibilidades de pensar la totalidad del sistema mundo, las que han sido negadas e inexistentes para la modernidad/colonialidad. En la afirmación del excluido, del oprimido, del sujeto de la diferencia colonial subalternizada se despliega un discurso que resignifica los sistemas de representación que nos habitan, los modos de comprensión que nos rigen, las prácticas de interrelación con nuestro entorno, el modo en que otorgamos valor a verdades y saberes construidos desde un locus de enunciación construido como referencia universal. El caso de Azkintuwe es una muestra de estas voces disidentes, disruptivas, creativas que buscan significar el mundo para resignificarlo, irrumpiendo la normalidad colonial por medio de hermenéuticas pluritópicas - en palabras de Mignolo (2003) - capaces de poner en jaque a la colonialidad y sus repertorios semióticos. Azkintuwe en la red digital es un proyecto decolonial en curso, enfrentado a la colonialidad del mundo global y el Estado-nación chileno y promoviendo un nuevo escenario de relaciones interculturales fundadas en el reconocimiento y validación de la diferencia etnocultural. Azkintuwe es un periodico digital que comunica desde la diferencia, interviniendo la semiosfera colonial a partir de la confrontación de sentidos y la propuesta de legitimar la identidad del excluido por medio de la validación y autoafirmación del ser como otredad. Los discursos analizados de Azkintuwe se enfrentan a la colonialidad del 
Estado-nación chileno y el sistema jurídico-judicial resignificando la noción de justicia, configurada dentro de la modernidad/ colonialidad como dispositivo de control y disciplinamiento de la población. De la justicia como acto punitivo, se piensa la justicia como reconstrucción del marco de relaciones interculturales entre el Estadonación chileno y el Pueblo Mapuche, enfatizando en la necesaria ruptura del modelo políticoadministrativo fundado en los relatos de la nación como unicidad y la pertenencia con la nación como máxima ética de los sujetos que habitan sus límites geopolíticos e imaginarios.

El análisis ha permitido observar una compleja estructura de significación respecto al tratamiento de la justicia/injusticia en el marco del conflicto entre Estado-nación y Pueblo Mapuche. Compleja, porque se evidencia una clara resignificación de estos conceptos en función del locus de enunciación desde el cual están siendo manipulados. Toda aplicación de justicia efectuada desde la institucionalidad hegemónica -entiéndase sistema jurídico-judicial y Estado-nación- es propuesta en términos de injusticia, respaldándose en factualidades (aplicación ley antiterrorista, procedimientos judiciales irregulares, informes internacionales en materia de Derechos Humanos) y evidenciando estrategias narrativas, discursivas, relaciones logico-semióticas y relaciones tetralémicas diversas en su estructuración. La justicia se convierte así en un dispositivo de la colonialidad que se ejerce sobre el Pueblo Mapuche en el marco del conflicto. El mapuche es clasificado como un enemigo de la nación chilena, un terrorista, que debe ser juzgado y sentenciado. Sin embargo esta lógica es confrontada $y$ resignificada en los disursos de Azkintuwe. Ahora bien, cuando la justicia es contruida desde el eje de la decolonialidad, el tópico adquiere sentidos asociados a las prácticas de liberación que efectúa el Pueblo Mapuche para combatir la colonialidad. En este sentido, la justicia se somete a una semiosis alternativa respecto a su diseño como norma/colonial. La justicia se presenta com un tópico asociado a la reivindicación (y no al delito), a la autonomía (y no al terrorismo), al diálogo intercultural (y no al multiculturalismo racial) y a la pluriversalidad como opción ( $\mathrm{y}$ no al universo colonial que habitamos).

En los textos analizados se observó que para el discurso construido por el periódico, ser mapuche es sinónimo de ser discriminado, en el sentido de ser reconocido como identidad aparte de la chilena. Es decir, para el Azkintuwe el chileno, con una identidad distinta a la propia mapuche-, reconoce al mapuche como otro diferente, pero de manera negativa, discriminándolo y segregándolo. Ahora bien, y respecto al autorreconocimiento como un sujeto distinto al otro - en este caso, al chileno- es de manera positiva, porque se resaltan valores como la heroicidad o la resistencia. En el discurso periodístico configurado por el periódico 
Azkintuwe también se puede apreciar una posición de poder de victimización del mapuche frente a un Estado chileno que viola la ley de igual libertad, que asegura un trato equitativo e integrador en la sociedad, por tanto, de esta manera se ejerce una violencia pasiva psicológica de un trato desigual, pero también una violencia física.

Así también, es posible identificar una posición de poder de heroicidad, lucha $\mathrm{y}$ resistencia en torno al mapuche que es consecuente y constante en su lucha de territorio y derechos.

En este sentido, según el discurso mediático del medio, para que exista justicia, debe existir un castigo en caso de hallar a los culpables de los delitos, y en el caso de no aplicarse dicha sanción, es porque entonces se asume de inmediato que no hay justicia, o sea, injusticia.

Ya hemos planteado anteriormente que según el imaginario configurado por el periódico Azkintuwe respecto a la justicia, ésta es insatisfactoria, y genera desconfianza y descontento entre miembros del pueblo mapuche.

\subsection{E1 discurso de la prensa: El discurso del diario El Austral de La} Araucanía

14 ROJAS, C.V.; et al: Discriminación Étnica En Los Tribunales Y En La Prensa De La Región De La Araucanía En Chile
El diario El Austral de La Arancanía utiliza la tribuna del género de opinión, en este caso, sus editoriales, para criticar, cuestionar y sugerir con respecto al conflicto mapuche en La Araucanía. Es así, que este periódico regional se ha encargado de construir socioimaginariamente al Pueblo Mapuche como una etnia conflictiva, la cual, no busca caminos de diálogo.

$\mathrm{Al}$ revisar minuciosamente cada texto (editoriales) se hace una clara mención al Pueblo Mapuche como una cultura que no busca el diálogo, ni el consenso, privilegiando muchas veces la violencia extrema para exigir sus demandas. Lo anterior se manifiesta claramente cuando este periódico, en reiteradas oportunidades, a través de sus editoriales, comenta con exactitud y detalle cada hecho de violencia que se vive en La Araucanía, como por ejemplo; quema de camiones en la carretera, toma de predios, quema de viviendas y casas patronales, violencia contra propietarios de los terrenos, entre otros.

Estadísticamente, podemos afirmar que del 100\% de las editoriales revisadas para esta muestra, en un $80 \%$ porciento encontramos argumentos y relatos con muchos detalles de los diferentes atentados que agricultores ha sufrido en zona. Existe una interpelación de este medio al Estado, al gobierno de turno, a la clase política, a 
los agricultores y a los propios mapuches, por no sentarse a conversar sobre el futuro que todos estos sectores quieren para la región, dejando atrás diferencias tanto ideológicas como políticas.

En esta construcción socioimaginaria, El Austral de La Arancanía, a través de sus editoriales apela a un discurso reflexivo por el futuro de la región, esto porque, el denominado conflicto mapuche en esta zona, ha provocado la notoria baja en las cifras de producción económica, todo ello, se debe a la inseguridad que presenta esta región del país en determinados sectores y lugares, provocando, que La Araucanía esté por debajo de los índices de producción, lo que conlleva mayor cesantía e inestabilidad laboral para la zona.

El medio de comunicación presenta al Pueblo Mapuche, en gran parte de sus relatos, como un pueblo violento, no abierto al diálogo, el cual utiliza la intimidación en su bandera de lucha por la reivindicación de tierras. Es más, esta violencia e intranquilidad social, ha producido que emprendedores de la zona (agricultores, dueños de tierras) no inviertan en la región por temor al clima sin paz que se viven en La Araucanía. Además, se plantea que la entrega de tierras al Pueblo Mapuche va a generar un retraso enorme para la producción agrícola en la región, ya que éstos, no poseen ni los recursos, ni los conocimientos y mucho menos la experiencia para trabajar la tierra desde una mirada más capitalista (producción al por mayor y entrega de productos a nivel nacional e internacional).

Otro de los imaginarios sociales que nos encontramos en estos discursos de opinión, tiene relación a la estigmatización que existe de determinados lugares (comunas) en La Araucanía, entre ellos; Ercilla, Collipulli y Chol - Chol. Esto, en gran parte se debe a que los hechos violentos (quema de viviendas, maquinarias) han ocurrido en estas zonas, lo cual, genera en la ciudadanía un clima de desconfianza y prejuicios, estigmatización que han tenido que sobrellevar, lo que ha provocado pobreza y cesantía en estos lugares. Por otra parte, en los discursos de opinión de este medio de comunicación se utiliza el término de "el mundo mapuche", segregando claramente entre ciudadanos mapuches y no mapuches, presentando a una cultura ajena, la cual, nos evoca a una etnia conflictiva, terrorista y no abierta del diálogo.

Otro de los términos que en reiteradas oportunidades sale a la palestra al momento de abordar el conflicto mapuche es "encapuchados armados", un concepto que designa a grupos manifestantes que expresan su descontento a través de la violencia a cara cubierta. Dicho término es utilizado en la mayoría de los relatos de opinión del Diario El Austral, lo que provoca en la opinión pública temor e incertidumbre, ya que existe un grupo terrorista que lucha por la 
reivindicación de tierras utilizando la extrema violencia.

Otro de los imaginarios sociales que encontramos tiene relación a la región de La Araucanía, una zona que para el resto del país es un lugar donde reina la intranquilidad social, la violencia y el conflicto, producto de la reivindicación Mapuche, etnia que no se siente parte de Chile y que lucha constantemente por la preservación de su cultura.

Para los discursos de opinión del Diario El Austral, los hechos y la significación que estos tienen son fundamentales, ya que por medio de estos relatos se hace una clara asociación entre lo que se hace (hechos de violencia) y el sentido que esto provoca (clima de intranquilidad social) en la ciudadanía. Por ello, en gran parte de estos relatos, encontramos que la pobreza en La Araucanía se debe, según este medio, al conflicto mapuche, porque éstos no trabajan la tierra. De la misma manera, encontramos discursos que apoyan la estigmatización en las zonas de conflicto, debido a que estos lugares continúan cercenados productivamente.

\subsection{E1 Discurso De Los Sujetos:} Redes Semánticas En Hombres Y Mujeres De Nivel Socioeconómico Medio Y Bajo En Temuco

16 ROJAS, C.V.; et al: Discriminación Étnica En Los Tribunales Y En La Prensa De La Región De La Araucanía En Chile redes semánticas. Este artículo pretende mostrar los resultados de la aplicación de una investigación usando las redes semánticas naturales en estudiantes de dos carreras de la Universidad de La Frontera para conocer las representaciones sociales que tienen respecto al denominado conflicto Estado chileno-Pueblo Mapuche usando cuatro frases-estímulo. Los resultados indican que existe una percepción de la situación en la que se resalta la situación de conflicto, con una mirada muy negativa con respecto al gobierno, el sistema judicial, y los medios de comunicación. Se discute las implicancias para el futuro desempeño profesional de estos estudiantes en caso de desenvolverse en un contexto laboral de intervención directa o indirecta en este conflicto.

Este estudio buscó sentar las bases para iniciar un debate serio e informado respecto a las representaciones sociales del conflicto Estado chileno-Pueblo Mapuche. Si bien presenta una serie de limitaciones (tamaño de la muestra, ubicación geográfica muy focalizada, todos los participantes provienen de la misma Universidad), es de esperar que permita dar pie a estudios más complejos respecto al tema, y especialmente lleve a la discusión respecto a la sociedad que queremos ser, y cómo podemos llegar a serlo. Si bien este conflicto es emblemático en Latinoamérica por su duración y relativa estabilidad a través del tiempo, está lejos de constituir una anomalía. Las 
comprensiones que nuestras sociedades han construido respecto a sus pueblos originarios han cambiado en las últimas décadas. Resulta imprescindible conocer las representaciones que se ha hecho de ellos, y que las políticas públicas respondan a las demandas de la ciudadanía en ese sentido.

\subsection{El Discurso De Los Sujetos: Las} Representaciones Sociales En Hombres Y Mujeres De Nivel Socioeconómico Medio Y Bajo En

\section{Temuco}

Para conocer las representaciones sociales sobre la relación entre el Estado y el Pueblo Mapuche en hombres y mujeres de la ciudad de Temuco, se aplicó una entrevista a una muestra de 80 adultos entre 25 y 35 años, agrupados según género, etnia (mapuche/no mapuche) y clase social.

Se aplicaron diferentes instrumentos de análisis del discurso para detectar el argumento que sostiene el discurso de cada sujeto junto con los macro-discursos ideológicos que habita. Estos instrumentos distinguieron las siguientes categorías: "rol medios de comunicación"; "Justicia/injusticia"; "relación Estado-Pueblo mapuche"; "Pueblo mapuche"; "actuación policial”. Los resultados muestran una concepción negativa generalizada tanto de la actuación de las instituciones estatales como de la labor informativa de los medios de comunicación al tratar con el pueblo mapuche. También se detecta un discurso mayoritario que reconoce injusticias históricas por parte del Estado hacia el pueblo mapuche. Esta investigación es parte de un proyecto de 3 años financiado por el Fondo Nacional de Desarrollo Científico y Tecnológico de Chile, Fondecyt número 1120904.

Para conocer las representaciones sociales sobre la relación entre el Estado y el Pueblo Mapuche en hombres y mujeres de la ciudad de Temuco, se aplicó una entrevista en profundidad a una muestra de 40 adultos entre 25 y 35 años, conformada por un total de 8 grupos de cinco personas agrupadas según el género, la pertenencia o no a la etnia mapuche y la clase social (media o baja). Se aplicaron diferentes instrumentos de análisis del discurso y se distinguieron las siguientes categorías: "Pueblo mapuche"; "rol medios de comunicación"; "Justicia/injusticia"; "actuación policial"; y "relación Estado-Pueblo mapuche". Los principales resultados arrojados muestran una concepción negativa generalizada tanto de la actuación de las instituciones estatales como de la labor informativa de los medios de comunicación a la hora de tratar con el pueblo mapuche. También se detecta un discurso mayoritario que reconoce injusticias históricas por parte del Estado hacia el pueblo mapuche. 
El desarrollo regional debe procurar un crecimiento más equilibrado de los distintos ámbitos de la vida social. Así, los aspectos culturales en este contexto son centrales, allí los discursos de género, los roles que desempeñan mujeres y hombres en la sociedad constituyen un eslabón fundamental para cimentar la calidad de vida de los habitantes de la zona, acorde a los valores democráticos e igualitario en boga en la cultura chilena (De Laudetis, 1989).

La cotidianeidad que viven las mujeres y hombres en la sociedad es el resultado de un modo de ver la realidad en la que la subjetividad participa de las representaciones sociales. Por un lado, lo cotidiano, como el subtexto del tejido de las acciones sociales, implica una relación del sujeto con sus representaciones. Berger y Luckman (1997), entienden la cotidianidad como el producto de un trabajo de determinaciones compartidas de lo que establecemos como la realidad y una vez hecho, deviene la realidad objetiva (da) en la que nos movemos. Lo cotidiano son siempre vivencias para un sujeto, que le asigna un significado de acuerdo al código de su colectivo o sociedad.

Lo cotidiano es significado como "en casa" donde no hay incertidumbre. Y por otro, el concepto de "norma" se entiende como una convención social que pretende regular la conducta de los sujetos. Es decir un parámetro que por ende, determina dicha conducta en un momento dado y espacio específico. La norma ha sido relacionada con el comportamiento prosocial, o por lo menos, uno de sus objetivos es instaurarlo en el sujeto como componente de su socialización (Cáceres, 2002).

Los resultados de la actual investigación provienen de la realidad nacional y de la realidad regional en que vive la sociedad chilena en su conjunto. La lectura que se tiene sobre el conflicto Estado-Nación y Pueblo Mapuche proviene exactamente de la realidad co-construida por el Estado y por los medios de comunicación nacionales. Lo que da paso a la construcción de representaciones sociales en cada individuo.

Cada situación de violencia vista en actos de reivindicación de derechos indígenas y/o las mostradas por los medios de comunicación genera en las personas una impresión de rechazo tanto a la violencia misma, como a los temas y sujetos involucrados en el conflicto.

Los estilos de ejercer la violencia han evolucionado en la actualidad. Históricamente el conflicto del Pueblo Mapuche ha estado presente en la realidad nacional, pasando de un conflicto de tierras, a un conflicto de Estado. Es así que la percepción actual que se tiene de la violencia puede entenderse desde esta realidad histórica y construida. 
En los resultados se aprecia que los sujetos en general tienen una visión negativa del Pueblo Mapuche, cuyos argumentos apuntan violencia a la que recurren estos para lograr sus demandas. Es interesante observar cómo esta concepción no sólo proviene de personas no mapuches sino que también de personas mapuches.

El rol que juegan los medios de comunicación en esta percepción es relevante al momento de observar cómo estos dan tratamiento a las noticias relacionadas con el conflicto, es decir, el tiempo que dedican, las imágenes que destacan y los discursos que validan públicamente. Sin embargo también existen las imágenes no mostradas y los discursos opacados. Es interesante además, el contraste existente entre las representaciones que se tienen sobre la policía y la institución policial, siendo ésta en muchos casos la causante de la violencia en las zonas de conflicto.

Los discursos se centran en: (a) el poder que tiene la institución sobre el actuar de cada policía, librando a estos de la responsabilidad individual de actos violentos, abuso de poder y de transgresiones a los derechos de las personas; (b) en como la policía es autora y responsable de generar e incentivar la violencia; y (c) cómo la policía es víctima de la violencia ejercida por mapuches. Como estos discursos son compartidos entre personas mapuches y no mapuches, se evidenció por un lado, el grado de compromiso de mapuches con las demandas indígenas y por otro el interés o desinterés de la temática indígena nacional por parte de no mapuches.

Sobre las representaciones centradas en los temas justicia e injusticia, se observa como mapuches y no mapuches apuntan a que la solución al conflicto indígena depende del Estado y no de la justicia como institución. Sin embargo, el tratamiento discursivo de gran parte de los conflictos mediatizados muestran cómo los actos de reivindicación son resumidos a un acto vandálico donde existen dos bandos: la policía, quien debe resguardar el orden y la paz, y los mapuches, quienes son los generadores de los disturbios y que deben ser controlados para que se restaure la tranquilidad. Dicho estado de tranquilidad debe ser logrado, tanto en la vida de las personas involucradas (ambos bandos), como en la vida de las personas no involucradas que de alguna manera se ven dañadas: vecinos, transeúntes, dueños de tierras, empresarios, trabajadores y telespectadores. Personas que dependiendo de su grado de adhesión a las causas del Pueblo Mapuche o a las del Estado, van socializando sus representaciones sobre el conflicto en sus relaciones sociales como en la familia, por lo tanto es en este proceso donde los medios de comunicación avalados por el Estado validan determinada información para ser 
compartida y luego socializada por quienes la aceptan como realidad.

Si se considera la adhesión a una causa ideológica y/o política como la aceptación de una realidad construida por determinados actores portadores de información, verdades y promesas, el trasfondo social del conflicto se puede acotar a que mientras más adeptos logre la causa mediatizada del Estado (bienestar, tranquilidad, orden, paz), menos seguidores tendrá la causa mediatizada mapuche (pobreza, rebeldía, terrorismo, violencia).

Se puede señalar que en el caso del discurso de la prensa, los periodistas escriben relatos "con su estructura, su orden, su punto de vista, sus valores. Los medios modelan, a la vez que reflejan la configuración y la expresión de la cultura, la política, la vida social" (De Fontcuberta y Borrat, 2006). Y, efectivamente, como consecuencia de lo anterior y si consideramos los elementos propios del proceso de producción y consumo, como las rutinas periodísticas, la estética del lenguaje multimodal y la apropiación del contenido mediático por las audiencias, "la noticia se transforma de esta manera en una tecnología, no sólo cognitiva, sino productora de lo real: es historia que crea historia" (Sodré, 1998).

\section{Algunas Consideraciones Finales}

A través de los medios, el Estado crea una imagen, un estereotipo, una representación del indígena que no es acorde con una sociedad de bienestar, más bien es una imagen que acerca al indígena a la barbarie, al estancamiento del progreso económico, a la pobreza, a la violencia desmedida e irracional, concepción que no es compatible con los valores de una democracia. Esto genera de cierta manera, una representación negativa de las personas mapuches (discriminación directa e indirecta por parte de la sociedad en general) y una idea de orden y seguridad (bienestar y tranquilidad nacional) que estos alteran.

Es una realidad, que ninguna persona está de acuerdo con las muertes de policías en servicio, asimismo nadie está de acuerdo con la muerte de personas mapuches. Sin embargo, el nivel de violencia policial y la militarización de sectores en conflicto sólo han demostrado la incapacidad de los distintos gobiernos de resolver de manera política y social una larga historia de injusticias y transgresión a los derechos humanos.

La gravedad del asunto es que la justicia (en tanto institución) no puede garantizar la justicia (como promesa y utopía) ni cualquier forma de verdad que no sea una tautología o tecnicismo; y, en consecuencia, lo único que debe 
garantizar, a saber el trato igualitario y no discriminatorio ante la ley, no lo hace. Por lo tanto, no queda más que infraestructura y burocracia.

Producir una sentencia nunca ha sido ni podrá ser un acto fuera de las condiciones ideológicas de la palabra y el sentido que desde ellas queremos otorgar a la realidad social y cultural en la cual y desde la cual se actúa. La realidad jurídico-judicial no es menos ideologizada que cualquier otro tipo de realidad construida socialmente. Por esta razón, el principal argumento es el derecho mismo (del tipo: "la ley lo establece así", "es el Estado de derecho", etc.), pero como éste ha sido social y políticamente construido en algún momento histórico (prueba de ello es su particularidad y modificaciones constantes según los distintos contextos) no es más que la conformación de una prisión desde el lenguaje, lo cual queda de manifiesto por el cada vez mayor descreimiento en el cual se encuentra la justicia en la sociedad; y la justicia no es más ni menos que la promesa, la utopía del "Estado de derecho", siempre pretendido y nunca logrado. Por lo mismo, la sociedad, a través del sentido común, muy lógico por lo demás, considera que no siempre a más derechos y leyes hay más justicia, sino que al contrario, parece haber menos justicia. En este escenario, los tribunales se especializan en producir juicios desde el derecho, pero no justicia. Nunca ha sido así.

\section{Referencias}

BERGER, P. y Luckmann, T. (1997). La construcción social de la realidad. Buenos Aires: Amorrortu.

CÁCERES, N. (2002). Representaciones sociales de la violencia y el conflicto en habitantes de la Comuna Uno de la ciudad de Santiago de Cali. Pensamiento Psicológico. Pontificia Universidad Javeriana Cali. Facultad de Humanidades y Ciencias Sociales. ISSN 1657-8961.

CORTE INTERAMERICANA DE DERECHOS HUMANOS

DE FONTCUBERTA, M. y Borrat, H. 2006: "Periódicos: Sistemas Complejos, Narradores en Interacción” Buenos Aires: La Crujía

De Laudeti, T. (1989). Tecnología de Género. Essays on Theory, Film and Fiction, London, Macmillan Press.

DEL VALLE, C. (2013). "Peritaje analíticodiscursivo sobre las evidencias de estereotipos, prejuicios y discriminación en los Tribunales de la Región de La Araucanía, Chile”, informe inédito, solicitado por la Federación Interamericana de Derechos Humanos para ser presentado a la Corte Interamericana de Derechos Humanos, Chile.

DEL VALLE et al. (2012-2015). "Medios de comunicación y poder: discursos de la Prensa y de 
sujetos adultos de la región de La Araucanía sobre justicia/injusticia en torno al Conflicto EstadoNación y Pueblo Mapuche". Proyecto financiado por el Fondo Nacional de Desarrollo Científico y Tecnológico, FONDECYT, número 1120904. Chile.

ITURRA, G. (2015). “Construcción de la noción justicia/injusticia en el discurso mediático del periódico Azkintuwe en el período 2003-2011”, Tesis de Magister en Ciencias de la Comunicación, Universidad de La Frontera.

MALDONADO, C. (2014). "Decolonialidad en las redes virtuales: El caso de Azkintuwe", Tesis de Doctorado en Comunicación y Periodismo, Universidad Autónoma de Barcelona.

PACHECO, S. (2015). "El imaginario social de la justicia en los diarios fundacionales de la Región de La Araucanía. La construcción de mito de La Araucanía", Tesis de Magister en Ciencias de la Comunicación, Universidad de La Frontera.

SÁEZ, C. (2013). "Imaginarios sociales sobre justicia/injusticia en torno al "conflicto estadonación y pueblo mapuche" en las editoriales del diario El Austral de La Araucanía durante 2003 2011”, Tesis de Magister en Ciencias de la Comunicación, Universidad de La Frontera.

SANHUEZA， C. (2014). "Representaciones sociales sobre la relación estado-nación y pueblo 22 ROJAS, C.V.; et al: Discriminación Étnica En Los Tribunales Y En La Prensa De La Región De La Araucanía En Chile mapuche en hombres y mujeres de Temuco", Tesis de Magister en Desarrollo Humano Local y Regional, Universidad de La Frontera.

SODRÉ, M. (1998). Reinventando la cultura: la comunicación y sus productos, Barcelona: Gedisa 